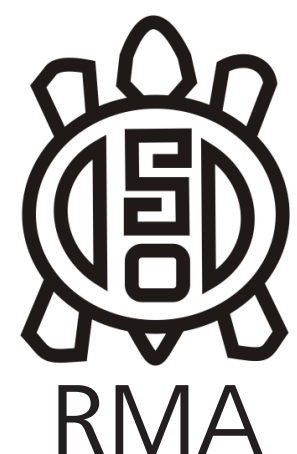

Arqueología

\title{
La arqueología desde el microscopio. Aportes interdisciplinarios de las diatomeas a las problemáticas arqueológicas
}

\author{
The archaeology from the microscope. Interdisciplinary contributions of \\ diatoms to archaeological problems \\ Lorena G. Grana
}

Universidad Nacional de Jujuy, Facultad de Ciencias Agrarias, Argentina. E-mail: lorenaggrana@yahoo.com.ar

\begin{abstract}
Resumen
Actualmente, las problemáticas arqueológicas generaran datos no solo desde la propia de la disciplina sino también desde teorías y metodologías provenientes de otras disciplinas. La Diatomología, una disciplina que forma parte de las Ciencias Biológicas, ha demostrado ser una importante herramienta para la Arqueología. El análisis de diatomeas ha sido aplicado en reconstrucciones paleoambientales, en el análisis de procesos de formación de sitio, en material arqueológico como el estudio de procedencia de materia prima para la cerámica, en el estudio del manejo prehistórico del agua y suelos, en los restos bioarquelógicos y, por último, en la conservación del patrimonio tangible. El objetivo del presente trabajo es realizar una revisión crítica de la evolución y estado actual de los análisis diatomológicos en cuestiones arqueológicas, principalmente incluyendo los estudios latinoamericanos. Para ello se propone discutir las potencialidades y limitaciones de estos estudios, con el fin de generar un puente conceptual que sea de utilidad para investigadores interesados en trabajar con estas líneas.
\end{abstract}

Palabras claves: Diatomeas; Procesos de Formación de sitio; Registro Arqueológico; Bioarqueología; Agricultura.

\begin{abstract}
Current archaeological problems need to generate data not only from the own discipline but also from the theories and methodologies of other disciplines. Diatomology, a discipline that is part of the Biological Sciences field, has proven to be an important tool for Archaeology. The diatom analysis have been applied in paleoenvironmental reconstructions, site formation processes analysis, the archaeological record such as the study of the provenance of ceramics, the study of prehistoric management of soil/water, in bioarqueological remains and, finally, in the conservation of tangible cultural heritage. The aim of this article is to present a critical review about the evolution and current condition of the diatom analysis in Archaeology, mainly in Latin American studies. We propose to discuss the potentialities and limitations of these studies, in order to offer a conceptual bridge that will serve researchers interested in working with these lines.
\end{abstract}

Keywords: Diatoms; Formation Processes; Archaeological Record; Bioarchaeology; Agriculture.

En las últimas décadas las investigaciones arqueológicas han generado datos desde enfoques interdisciplinarios para responder a diversas problemáticas. En este sentido, el arqueólogo debe ir más allá de las descripciones de los atributos del material arqueológico (color, forma, tecnología, medidas, decoración, etc.), por lo que debe valerse de teorías y metodologías provenientes de otras disciplinas para interpretar nuevos datos. Por consiguiente, una de las particularidades de la Arqueología es promover la adopción de los saberes generados en otras disciplinas científicas, adecuándolos a las preguntas arqueológicas, e incluso en algunos casos impulsar el desarrollo de nuevos conocimientos, generando de este modo una relación retroalimentaria entre las disciplinas.
A lo largo de su desarrollo disciplinar la Arqueología ha creado enormes rompecabezas de información procedente de múltiples disciplinas que, sin embargo, requieren de una articulación y contrastación cuidadosa para minimizar los riesgos generados por los límites disciplinarios. Esto implica que los investigadores dominen no sólo el lenguaje y las metodologías de su campo disciplinario sino también el de otras disciplinas. Por este motivo, los trabajos de síntesis y artículos de revisión cumplen un rol importante para desarrollar este "lenguaje en común", debido a que facilitan el acceso de forma organizada a los conocimientos existentes en una temática tanto para los investigadores de la misma disciplina como de otras. 
Una de las relaciones interdisciplinaria más tradicionales que ha generado la Arqueología con otros saberes ha sido con las Ciencias Biológicas, de donde provienen diversas teorías y métodos que han sido adaptados a las problemáticas arqueológicas. La Diatomología se ubica dentro de la Ficología, que es una de las disciplinas de la Botánica (rama de las Ciencias Biológicas). Principalmente, uno de los objetivos de esta subdisciplina es estudiar las diatomeas, las cuales son microorganismos unicelulares, algas eucariotas, diploides y fotosintéticos, pertenecientes a la clase Bacillariophyceae (Round et al. 1990). Las diatomeas pueden vivir en ambientes muy diferentes con diverso tenor salino (aguas dulces a hipersalinas), estado trófico, pH (ácido a alcalino) y temperatura (condición de hielo a aguas termales). Además, uno de los rasgos distintivos de estos microorganismos es que su cubierta celular está conformada por sílice $\left(\mathrm{SiO}_{2} \mathrm{nH}_{2} \mathrm{O}\right)$, lo que le permite tener un alto grado de preservación de las valvas que la conforman (Battarbee 1986; Round et al. 1990; Cooper et al. 2010; Julius y Theriot 2010). Por consiguiente, la ubiquidad de las diatomeas y su capacidad de responder rápidamente a cambios físico- químicos en su medio, las convierten en valiosos indicadores biológicos y en una herramienta de alta utilidad para diversas disciplinas como la Limnología, la Paleolimnología, la Paleooceanología e incluso la Arqueología.

Actualmente, existen algunos trabajos que han compilado las aplicaciones de los análisis de diatomeas en las discusiones arqueológicas demostrando su utilidad (Mannion 1987; Battarbee 1988; Cameron 2007; Juggins y Cameron 2010). Sin embargo, estas recopilaciones concentran los trabajos arqueológicos provenientes principalmente de regiones del Norte de Europa o de América, dejando de lado las aplicaciones que se han realizado en otras regiones como Latinoamérica. Hasta el momento, se registra una sola recopilación de este tema que incluye a los estudios latinoamericanos (Martínez Macchiavello et al. 1999), pero se encuentra desactualizada ya que en la última década ha incrementado las aplicaciones de las diatomeas. Por este motivo, en el presente trabajo se propone generar un resumen actualizado de las aplicaciones de las diatomeas en problemáticas arqueológicas, junto a un análisis crítico para discutir sobre sus potencialidades y limitaciones. El objetivo último es generar un puente conceptual que sirva tanto para arqueólogos como biólogos, u otros investigadores interesados en trabajar con esta línea de investigación.

\section{Breve Historia}

El estudio de las diatomeas tiene una vieja tradición en las ciencias occidentales. En el siglo XVIII aparecen las primeras descripciones y dibujos de especies de diatomeas, impulsadas por el progreso de las tecnologías ópticas, como el microscopio (Round et al. 1990). Sin embargo, recién a mediados de XIX y principios del $X X$, comienzan a crecer sistemáticamente los estudios de diatomeas generando importantes colecciones y monografías taxonómicas principalmente gracias a los aportes de los investigadores y naturalistas como $\mathrm{C}$. Ehrenberg, O. Müller, F. Kutzing, A. Grunow, P. T. Cleve, J. Tempère, J. Frenguelli, F. Hustedt y G. Deflandre, entre otros, quienes son pioneros de la Diatomología (Vouilloud 2003). Este período se caracteriza por el afán del descubrimiento y de las grandes exploraciones para identificar nuevos taxones, lo que llevó a estudiar materiales de diversos orígenes (plantas, suelos, sedimentos lacustres, sedimentos marinos, turbas e incluso muestras de guano) provenientes de numerosas regiones del mundo. De este modo, se describieron una gran cantidad de géneros y especies.

Para la mitad del siglo XX, la Diatomología comienza a cambiar su perspectiva tradicional hacia un enfoque orientado a responder preguntas fisiológicas, bioquímicas, genéticas, iniciando también relaciones con otras disciplinas como la Limnología, la Paleontología, la Ecología y la Arqueología, entre otras. Además, las investigaciones comienzan a recopilar cada vez más datos sobre las afinidades ecológicas de las especies en relación al hábitat, pH, nutrientes, salinidad, saprobiedad, etc. Por lo tanto, en base a la gran cantidad sistemática de datos ecológicos y taxonómicos de las especies, comienzan a usarse como bioindicadores de calidad de agua y bioproxy para las reconstrucciones paleoambientales de los cuerpos de agua (Battarbee 1986; Smol 2009). También, se evalúa su fidelidad composicional y ambiental desde una perspectiva de la Tafonomía actualística, experimental y comparativa (Hassan et al. 2008, Flower y Ryves 2009; Ryves et al. 2009; Hassan 2015). Estas tendencias de investigación continúan intensificando y complejizándose hasta la actualidad.

La relación interdisciplinaria entre la Diatomología y la Arqueología es de larga data. Los primeros análisis de diatomeas en problemáticas arqueológicas se registran al principio del siglo XX. A modo de ejemplo, se reconoce el trabajo de Patrick (1938), quien desde la evidencia de las diatomeas plantea una reconstrucción ambiental local de los sitios Clovis, además genera inferencias cronológicas para estos sitios en base a comparaciones de las especies halladas en otros registros. En Latinoamérica, se puede resaltar el trabajo de Frenguelli (1940), que a través de un análisis geomorfológico, geológico y diatomológico, estudia el origen de unos montículos asociados a ocupaciones arqueológicas en la llanura de Santiago del Estero (Argentina). A mediados de la década de los setenta y hasta los noventa, se inicia el momento de mayor auge de los análisis diatomológicos para responder preguntas arqueológicas a nivel mundial. Estos son aplicados a una gran diversidad de problemáticas que abarcan un amplio rango de escalas espaciales y temporales, demostrando de este modo la alta utilidad como herramienta para las 
investigaciones arqueológicas. Y en los últimos años, junto a otros microfósiles, ha incentivado el desarrollo de investigaciones multidisciplinarias (Korstanje y Babot, 2008).

\section{Distintas aplicaciones en problemáticas arqueológicas}

Las problemáticas más trabajadas en Arqueología mediante el análisis diatomológico han sido en las reconstrucciones paleoambientales tanto a escala regional como local, en los análisis de fuentes de arcilla para estudios de proveniencia y en los análisis de procesos de formación de sitio. En los últimos años se fue ampliando el rango de temas incorporando otros, como estudios particulares en diversos materiales arqueológicos, incluyendo restos bioarqueológicos, y el estudio del manejo de suelos y/o agua. Sin embargo, las aplicaciones de las diatomeas tienen potencialidades y limitaciones que son necesarias conocer antes de seleccionar este proxy para una investigación. Además, hay que considerar la gran variabilidad de enfoques que existen en su empleo, ya que dependiendo de éste se generan distintos resultados o grados de resolución a las conjeturas.

Reconstrucciones paleoambientales e impacto cultural en el ambiente

Entre los bioproxies más comúnmente utilizados, las diatomeas son consideradas como excelentes indicadores paleolimnológicos ya que son abundantes en los cuerpos de aguas. Además poseen una gran variedad de estrategias adaptativas $y$, como mencionamos anteriormente, su corto ciclo de vida les permite responder rápidamente a los cambios ambientales, considerándoselas como un proxy con alta sensibilidad al cambio ambiental (Cooper et al. 2010). Por otro lado, es necesario remarcar que las reconstrucciones ambientales han sido líneas de investigación de gran interés para la Arqueología, ya que una de las metas de la disciplina es entender cómo las sociedades se adaptan, conciben y afectan a los ambientes en que viven.

Muchas veces los modelos ambientales generados desde las Geociencias entran en conflicto con las preguntas arqueológicas, porque estos trabajan con escalas de resolución u objetivos propias de sus disciplinas (ej. Geología, Palolimnología, etc.), que no siempre son acordes a la Arqueología. Por lo que genera la necesidad de remodelar o elaborar modelos paleoambientales acordes a las problemáticas arqueológicas (Dubois 2006; Gutiérrez et al. 2011, Morales 2011, Grana et al. 2016). Estos modelos se construyen dentro de proyectos multidisciplinarios o interdisciplinarios con biólogos, geólogos, paleontólogos, palinólogos, entre otros, donde el arqueólogo cumple un rol participativo en la formación de los datos e interpretaciones paleoambientales. Ahora bien, los modelos paleoambientales generados desde las diatomeas han sido aplicados de forma muy variable dentro de las investigaciones arqueológicas. En relación a la escala en que se orienta la reconstrucción paleoambiental, estas investigaciones pueden ser agrupadas en dos grandes grupos.

El primer grupo estaría conformado por las investigaciones arqueológicas que trabajan a una escala regional, por lo que se focalizan en reconstruir la evolución de la dinámica de los ambientes fluvio-lacustres de la región estudiada para contextualizar o correlacionarlos con alguna problemática en particular (poblamiento, cambios sociales, explotación del paisaje, etc.). Incluso, en problemáticas con amplio rango cronológicos, como el estudio sobre los patrones de uso y explotación de humedales por parte de los homínidos entre el Plioceno y Pleistoceno (Ashley et al. 2009), debido a que las diatomeas tienen un registro fósil extensivo que inicia en el Cretáceo tardío. Las diatomeas dentro de este grupo pueden ser analizadas como parte de los estudios estratigráficos, donde se observa la geometría, las estructuras sedimentarias y el contenido biológico de los estratos. Por ello los depósitos diatomológicos tan sólo son identificados y descriptos, para ser interpretados sedimentológicamente a través de facies sedimentarias (Dubois 2006; Tchilinguirian y Olivera 2010). Otro enfoque, es el estudio particular de la composición del ensamble de diatomeas, donde se obtienen las abundancias relativas de las especies, dominancias de las especies y las afinidades ecológicas, bajo los fundamentos y metodologías de la Paleolimnología. De esta forma, se generan reconstrucciones cuantitativas o semicuantitivas de los cambios paleohidrológicos de los sistemas hídricos estudiados e infieren las condiciones paleoambientales/ paleoclimáticas (Owen et al. 1983; Blasi et al. 2005; Ognjanova-Rumenova 2008; Castiñeira Latorre 2009; Morales 2011; Fernández 2012; Grana 2013; Grana et al. 2016; Inda et al. 2016, entre otros).

El segundo grupo, está formado por las investigaciones que se orientan a realizar las reconstrucciones ambientales a escala local para establecer relaciones entre el ambiente y la ubicación de los sitios. Principalmente, se centra en el análisis de secuencias sedimentarias naturales para caracterizar ambientes puntuales cercanos a los sitios arqueológicos $y$, de este modo, entender el desplazamiento de los sitios en el paisaje (Gearey et al. 2011; Weide et al. 2017). Aunque, en otras investigaciones la escala local puede involucrar tan sólo el análisis de las condiciones microambientales dentro de los propios sitios arqueológicos, por lo que las muestras analizadas provienen de la secuencia sedimentaria propia de los sitios arqueológicos (Bonomo et al. 2009; Martínez et al. 2014; Colobig et al. 2016). Generalmente, estas últimas investigaciones se enfocan desde un análisis multiproxy, donde las diatomeas forman parte de un dato complementario dentro de los resultados, considerando sólo una cuantificación en base a presencia o ausencia. Las investigaciones realizadas en las sierras de Tandilia (Argentina) tienen este enfoque ya que utilizan el análisis 
de las diatomeas junto a otros restos biosilíceos (fitolitos) para reconstruir las condiciones microambientales de los sitios (Hassan 2012; Martínez et al. 2014; Colobig et al. 2016).

Por último, dentro de esta gran temática y a escala local también se han utilizado las diatomeas para identificar y evaluar el impacto cultural de diversas sociedades en los ambientes del pasado. Por ejemplo, se han utilizado a las diatomeas para identificar y reconstruir los impactos prehistóricos de la agricultura sobre los lagos (Bradshaw et al. 2006; Lozano-García et al. 2007; Inda et al. 2016). También las diatomeas han sido aplicadas para analizar el impacto que generan los asentamientos y actividades de cazadores-recolectores en los ecosistemas acuáticos, como las actividades llevadas a cabo por los balleneros Inuit prehistóricos en Mar Ártico (Hadley et al. 2010).

Estas temáticas y sus escalas de análisis no son incompatibles, y en algunas investigaciones pueden utilizarse de forma complementarias. El caso de Ognjanova-Rumenova y Zaprjanova (1998) es un ejemplo ya que desde el análisis de diatomeas en un perfil estratigráfico de un sitio arqueológico costero de Bulgaria determinan los cambios locales en el nivel del mar $y$, al mismo tiempo, el impacto de las actividades agrícolas prehistóricas en el ambiente durante el Calcolítico y la temprana edad de Bronce. En relación a la escala del modelo paleoambiental, algunas investigaciones utilizan ambas escalas para sus interpretaciones, como es el estudio realizado por Hassan (2012) en la región de la sierra de Tandilia (Argentina), donde analiza la composición florística de diatomeas proveniente de testigos de sedimentos de tres sitios arqueológicos, y en base a los resultados infiere no sólo las condiciones ambientales locales de los sitios, sino también discute su significado paleoambiental a nivel regional durante el Holoceno.

\section{Proceso de formación de sitio}

Para reconstruir con exactitud las actividades humanas del pasado, es un prerrequisito entender los procesos de formación que intervienen en los depósitos de los sitios arqueológicos. Los sedimentos recuperados de las excavaciones de los sitios brindan la información sobre estos procesos a través de diversos análisis, como químicos, físicos y biológicos (ej. larvas de insectos, roedores, anfisbénidos, diatomeas, etc.) (Mannion 1987; Kligmann 2009).

Las diatomeas también han sido utilizadas para determinar la génesis y la evolución de los montículos prehispánicos conocidos como "cerritos" en las tierras bajas del sureste de Sudamérica (Castiñeira et al. 2013). Del mismo modo, se han utilizado las diatomeas dentro de análisis micromorfológicos para analizar sitios particulares, como los que se generan por las acumulaciones antrópicas de valvas de moluscos y demás restos faunísticos, conocidos como Sambaqui (en Brasil) o concheros (en el resto de Latinoamérica) (Villagran et al. 2009).

Algunas investigaciones se centran en el análisis de sedimentos arqueológicos para ver alteraciones o modificaciones postdepositacionales sobre el registro arqueológico. Por ejemplo, la identificación de diatomeas en sedimentos provenientes de un alero en Patagonia, permitió identificar la presencia de agua en el sitio e inferir cambios de $\mathrm{pH}$ posterior a las ocupaciones prehispánicas, lo que actuó como agente corrosivo del material lítico (Fernández y Salemme 2012; Lynch et al. 2015). Otro caso, es la investigación desarrollada por Fugassa y colaboradores (2011) donde analizan sedimentos arqueológicos provenientes de los forámenes sacrales de esqueletos humanos. En estos sedimentos los investigadores recuperaron invertebrados y una gran cantidad de microfósiles, como diatomeas y rotíferos, lo que infirieron que el esqueleto se depositó inicialmente en un ambiente acuático o que posterior a su depositación, el sitio estuvo inundado. Estos casos demuestran la importante información que se puede obtener para interpretar los procesos de formación del depósito.

Dentro de esta temática, también se han estudiado las diatomeas en sedimentos arqueológicos para identificar áreas diferenciadas de actividades antrópicas en los sitios. Kligmann y colaboradores (2013) a través de las diatomeas provenientes del sitio El Zorro (Catamarca) establecen que el principal agente de transporte de estas algas fue antrópico, debido a que identifican una significativa diferencia en las densidades de valvas, tipos de géneros y fragmentación de valvas entre las muestras de sedimentos analizadas. Por lo que junto a otras líneas de evidencia, como la cerámica, las autoras infieren que ciertos sectores del sitio estuvieron destinados a actividades antrópicas particulares como la cocción o el almacenaje de líquidos, lo que explicaría la gran presencia de las valvas de diatomeas.

\section{En material arqueológico}

En lo últimos tiempos, los análisis de microfósiles en diversos materiales arqueológicos han tenido un alto impacto en las investigaciones arqueológicas. Las diatomeas no han estado excluidas de esta tendencia. Principalmente, se utilizaron las diatomeas para estudiar la procedencia de las materias primas de la cerámica. Esto se inicia con la Arqueología Escandinava en la década de los setenta, con el objetivo de identificar si las fuentes de arcilla provenían de la zona costera o del interior de la región, ya que las diatomeas marinas son muy distintas a las especies continentales (Håkansson y Hulthén 1988). También, estos análisis se utilizaron para inferir intercambios sociales a través de la correlación entre ensambles de diatomeas y estilos cerámicos (Alhonen et al. 1980; Wallis et al. 2014). Con menor aplicación se ha utilizado para ver la procedencia de ladrillos de adobe (Flower 2006). 
En Latinoamérica, se registran varios trabajos de este estilo, principalmente en Argentina, donde los estudios de procedencias de cerámica se centraron en Chaschuil (Catamarca), en Susques (Jujuy) y en el Delta Paraná e Isla García (Santa Fe- Buenos Aires) (De la Fuente 2002; Solá y Morales 2007; Capdepont y Bonomo 2010). También se registra una investigación con este fin, desarrollada en México (Obando et al. 2011). El principal potencial de las diatomeas para estos estudios se encuentra en que las fuentes de las materias primas para la manufactura de la cerámica provengan de ambientes con altos contrastes ecológicos entre sí. Por ejemplo, Solá y Morales (2007) estudia un tiesto estilo incaico hallado en un sitio de la puna jujeña (Argentina), y a través de la identificación abundante de un diatomea interpreta que tiene un origen alóctono. Las valvas reconocidas en el corte delgado del tiesto fueron determinadas como Cyclostephanos andinus (Theriot, Carney \& Richerson) Tapia, Theriot, Fritz, Cruces et Rivera. Esta especie es planctónica y vive en cuerpos de agua muy profundos, los cuales son poco frecuente en el Noroeste Argentino, lo que le permite establecer a los autores que la pieza analizada provino de alguna zona de los andes centrales, donde hay lagunas con las condiciones ecológicas requeridas por dicha especie.

Sin embargo, hay que tener ciertas precauciones con estos tipos de estudios, ya que tienen limitaciones importantes. Por ejemplo, durante la etapa de producción de la cerámica pueden existir distintos momentos donde se incorporen valvas que no necesariamente provienen de las fuentes de materias primas, también puede existir mezcla de recursos (agua, arcilla, arenas etc.) que interfieran en la identificación de una fuente específica, y por último la fragmentación y/o disolución de las valvas que suele generarse durante el momento de producción (amasado, temperatura de cocción etc.) (Gibson 1986; Håkansson y Hulthén 1986; Battarbee 1988; Kligmann y Calderari 2012). También es necesario tener en cuenta qué tratamientos se emplean para obtener las valvas de las muestras de cerámica. Generalmente, se emplean tres métodos para estos estudios. Dos métodos se centran en el tratamiento específico del tiesto para poder obtener las valvas, lo que incluyen la aplicación de métodos físicos, como la trituración del fragmento, y/o la aplicación de métodos químicos, como la disolución por medio ácido (De la Fuente 2002). El tercer método no implica un tratamiento específico para la obtención de valvas sino que se analiza directamente por medio de los cortes delgados realizados en los tiestos para los análisis petrográficos. Por lo general, estos estudios implican tanto análisis biológicos como mineralógicos de los tiestos (Solá y Morales 2007; Capdepont y Bonomo 2010; Obando et al. 2011).

Con la misma óptica, se ha aplicado el análisis de diatomeas en pigmentos arqueológicos utilizados en contextos funerarios de Calama y Chimorro (Chile) para identificar las procedencias del mineral que conforma los pigmentos usados sobre las momias (Olgade et al. 2015).

Otras aplicaciones de las diatomeas para estudios en cerámica son los análisis que se realizan en los residuos adheridos a la superficie de los objetos para inferir las actividades desarrolladas con ellos. En general, este enfoque implica investigaciones que abarcan análisis de múltiples microfósiles, donde el énfasis en las diatomeas se centra en identificar su presencia/ausencia en las muestras, y las interpretaciones se realizan en conjunto con todos los proxies analizados (Coil et al. 2003). Es el caso de los análisis realizados en ánforas ibéricas, donde a través de raspados de las paredes de estas cerámicas se obtuvieron residuos que permitieron identificar la presencia de almidones, diatomeas y restos de levaduras, e inferir que estas vasijas fueron utilizadas para la fermentación de bebidas alcohólicas y no para otro tipo de alimentos fermentados (Juan-Tresserras 2000). Por otro lado, algunos estudios van más allá de la presencia de valvas y se focalizan en la identificación taxonómica de las diatomeas halladas, lo que le permite al investigador generar deducciones más específicas. Entre otras, está la investigación realizada por Beltrán de Heredia Bercero y colaboradores (2006) que en base al análisis de residuos obtenidos de vasijas ibéricas, hallaron una especie de diatomea marina que indicaría que las vasijas contenían sales o agua marina, esto les permitió inferir "recetas culinarias" para la preparación de vinos, ya que las sales suelen utilizarse como un estabilizante en la fermentación del vinos. En Latinoamérica, estos estudios se han aplicado no sólo en residuos en tiestos de cerámica (Gasparotti y Haros 2015; Colobig y Ottalagano 2016; entre otros) sino también, en residuos provenientes de cucharas de cerámicas (Bonomo et al. 2012), pipas (Planella et al. 2012; Belmar et al. 2016) y tabletas de arcilla (Tuji et al. 2014).

Por otra parte, se han estudiado los residuos adheridos en otros materiales como en artefactos líticos de molienda, donde la presencia de diatomeas y otras partículas biosilíceas han sido utilizadas para identificar los procesamientos de vegetales con aditamento de agua (Babot 2004; Caria et al. 2011). También se han analizado las diatomeas adheridas en filos de instrumentos líticos provenientes de sitios arqueológicos de Australia, para inferir si se utilizaron para la cosecha y/o procesamiento de plantas acuáticas (Robertson 2006). Sin embargo, este último autor remarca la importancia de conocer bien los informes de excavación para contextualizar los hallazgos y los tratamientos post-excavación (ej. si fueron lavados u obtenidos por zarandas húmedas) ya que estos pueden interferir en las inferencias.

\section{Restos bioarqueológicos}

En medicina forense legal es común utilizar el test de diatomeas en tejidos cadavéricos para diagnosticar muertes por sumersión, ya que cuando una víctima se 
ahoga inhala agua y con ello incorpora las valvas y los fragmentos de diatomeas a la vía respiratoria. Estas pueden pasar a la circulación general a través de la ruptura de los alveolos del pulmón, y depositarse luego en los órganos y en la medula ósea. Por lo que el test tiene un importante valor para las inferencias forenses actuales (Maidana 2013). Siguiendo este enfoque, aunque de forma menos recurrente, algunos arqueólogos han aplicado el test de diatomeas para diagnóstico de muerte por sumersión en esqueletos arqueológicos. Se puede citar el estudio realizado en el material bioarqueológico del Neolítico tardío en Suecia, donde los investigadores analizaron las diatomeas provenientes de las muestras internas y externa de un hueso largo entero (húmero) y de la matriz sedimentaria. Las comparaciones de las comunidades de diatomeas halladas en las muestras más el contexto arqueológico, les permitió a los investigadores inferir que el individuo murió ahogado (Carlie et al. 2015).

Otras aplicaciones de los estudios diatomológicos en temáticas bioarqueológicas es el análisis de valvas proveniente de los cálculos dentales, que junto a otros microproxies (fitolitos, almidones, etc.), son considerados como buenos bioindicadores de las prácticas de subsistencia, ya que estos permiten generar inferencias sobre el tipo de alimento, la calidad del agua y tipo de fuente hídrica consumida en la ingesta alimenticia (Vargas Afonso 2007; Dudgeon y Tromp 2014; Vásquez Sánchez et al. 2014; Ramírez Ríos et al. 2008). También se han analizado las diatomeas conservadas en los coprolitos para estudiar la subsistencia humana, ya que presentan buena conservación. Por ejemplo, Flores Caballero (2014) identifica diversas diatomeas marinas en coprolitos humanos de un sitio prehispánico en Áspero (Perú) e interpreta que la presencia de estas especies indica el consumo de peces marinos. Por otro lado, el análisis de diatomeas en estos restos pueden indicar la estacionalidad en el uso de los recursos consumidos y por ende la estacionalidad de la ocupación, sobre todo si en la región los cambios estacionales son muy marcados, ya que como se mencionó anteriormente, la composición florística de diatomeas responde a estos cambios ambientales (Horrocks et al. 2002)

\section{Manejo de agua y suelos}

Los análisis de las diatomeas han sido de gran utilidad en problemáticas sobre la agricultura arqueológica. Por lo general, estos estudios parten desde un enfoque de multiproxy y las diatomeas son utilizadas para entender el manejo de los suelos y el riego en los espacios productivos (campos de cultivos, terrazas, etc.) (Horrocks et al. 2002; Korstanje 2005; Trombold e Israde-Alcantara 2005; Korstanje y Cuenya 2010; Zucol et al. 2012; Sánchez et al. 2013, entre otros). Esto se debe a que estas algas junto a las espículas de espongiarios y los estomatocistes de crisostomatáceas son interpretados como indicadores de humedad, por lo que muchos autores consideran su presencia como evidencia de riego o abono en campos de cultivos. También se ha utilizado a las diatomeas para analizar el manejo de suelos/turberas, como es el caso de las construcciones vikingas donde generan el material de construcción a partir de "ladrillos" de turba y el análisis de las especies ha permitido no sólo identificar estas estructuras arqueológicas, sino también identificar estos sectores de manejo de turba (Barthust et al. 2010). Otro caso de manejo de suelo, se registra en Nueva Guinea, donde esta línea de evidencia se la ha utilizado para la identificación y caracterización de los sectores de pantanos destinados a ser praderas antropogénicas para cultivos. Este manejo de suelo está asociado a una antigua tecnología agricultura conocida como Kuk Swamp (Denhman et al. 2009)

Otros autores han utilizado a las diatomeas como buenos bioindicadores para localizar y caracterizar distintas estructuras hídricas arqueológicas, mostrando su alto potencial para el estudio del manejo de agua. Por ejemplo, han demostrado ser de alta utilidad para identificar canales arqueológicos prehispánicos en la puna Argentina (Grana et al. 2014). Por otro lado, Cameron (2007) ha demostrado que estudiando las diatomeas provenientes de acequias romanas-británicas se puede obtener información sobre la corriente de agua, la influencia de la marea e identificar las actividades de mantenimiento. Dentro de esta línea, se encuentra la investigación presentada por Beneš y colaboradores. (2002), quienes se centran en el estudio de acequias de drenaje de un foso medieval de una antigua fortificación en Praga (Rep. Checa), demostrando la utilidad de las diatomeas para analizar la evolución hídrica de la fosa y los pozos medievales, así como también la identificación en el incremento de la contaminación del agua a través del tiempo.

Por otra parte, se ha utilizado a esta microalga junto a otros materiales biológicos, como macroalgas, esporas, briofitas y plantas acuáticas, para obtener dataciones del radiocarbónica de canales prehispánico, ya que estos organismos quedan atrapados en las capas de travertinos, cuando se precipitan junto a los carbonatos $\left(\mathrm{CaCO}_{3}\right)$, conformando las cubetas fosilizadas de evidentes canales prehistóricos (Winsborough et al. 1996). Por último, también se han analizado las diatomeas de otras estructuras hídricas, como los pozos de agua arqueológicos, mostrando su utilidad tanto paleoecológica como sedimentológica para la caracterización de estas evidencias (Meltzer y Collins 1987; Neely et al. 1995). En resumen, lo estudios presentados demuestran el gran potencial de las diatomeas tanto en la identificación de las estructuras hídricas como en la evolución del cuerpo de agua que contiene.

\section{Conservación del Patrimonio Arqueológico}

El estudio de la comunidad de diatomeas junto a otras algas y cianobacterias ha estado orientado a determinar el deterioro generado por las patinas de algas, ya que estas 
producen procesos de biotransferencia y ficodeterioro físico en las estructuras y esculturas arqueológicos e históricas. Principalmente, estos estudios han sido aplicados en monumentos y fuentes de agua medievales y romanas de Europa, donde se analizó la biomineralización producida por algunas diatomeas. Por ejemplo, el género Gomphonema Ehrenberg presenta especies epifitas, o sea viven adheridas a un sustrato, para ello genera tubos de mucilago y así generan colonias. Estos mucilagos forman costras de minerales en las superficies adheridas por lo que deteriora las paredes de las estructuras (Bolívar y Sánchez-Castillo 1997; Peraza Zurita et al. 2005). Entender el tipo de diatomea que puede estar afectando al patrimonio arqueológico permite elegir la mejor estrategia para la conservación.

\section{Algunas precauciones generales}

Las investigaciones resumidas en este trabajo muestran el alto potencial que tiene el empleo del análisis de las diatomeas para las discusiones arqueológicas, no obstante, como ha sido mencionado anteriormente, es necesario tener en cuenta algunas cautelas a la hora de realizar inferencias. En líneas generales hay cuatro recaudos que deben ser consideradas en todas las temáticas: contaminación, tafonomía, metodología y taxonomía.

En relación a la contaminación es necesario tener información certera sobre la proveniencia de las valvas que se analizan, ya que al ser organismos microscópicos la contaminación puede ser alta. Principalmente, podemos resaltar dos momentos críticos de contaminación, uno es durante el momento de obtención de las muestras y otro durante el tratamiento y/o conservación de las mismas. La disminución de la contaminación dependerá del material que estemos analizando. Por ejemplo, en las muestras provenientes de sedimentos las precauciones de contaminación durante el muestreo pueden disminuir si se siguen los criterios propuestos en las ciencias ambientales (limpieza del perfil antes del muestreo, toma de muestras del perfiles de abajo hacia arriba, etc.) (Battarbee 1986). En relación a los sedimentos arqueológicos proveniente de sitios o estructuras agrícolas, varios investigadores han recomendado analizar a modo de control una muestra lejana al sector estudiado, para poder comparar esa muestra con las proveniente del sitio (Kligmann et al. 2013).

Con respecto al material arqueológico o bioantropológico, es necesario garantizar que la obtención de las valvas provengan del material que queremos analizar y no de otros medios, como puede ser la matriz sedimentaria o las valvas de la pasta de vasijas para el caso de los análisis de los residuos adheridos. Para evitar interpretaciones erróneas algunos autores proponen ciertas precauciones metodológicas. En el caso del estudio de las diatomeas adheridas a cerámicas u otros objetos, se postula realizar dos raspados como mínimo, uno superficial para eliminar cualquier adhesión que provenga del contacto con los sedimentos arqueológicos y otro más intenso que permita recuperar los taxones adheridos en los poros del material analizado pero sin dañar el objeto (Babot 2004; Zucol et al. 2008). En otros materiales, como coprolitos, se propone remover las capas externas del material que están en contacto con sedimentos que pueden generar contaminación, y analizar sólo las muestras que provengan del material interno (Wood y Wilmshurst 2016). El análisis de los sedimentos de la matriz sirve como control para identificar el conjunto de microfósiles depositados de forma natural en el suelo y diferenciar aquellos asociados culturalmente al contexto de uso (Planella et al. 2012). Por ello, es necesario planificar un muestreo sistemático de los sedimentos durante la excavación, principalmente aquellos que están en contacto con el material arqueológico que será estudiado. Sin embargo, no siempre se cuenta con estas muestras, por lo que algunos investigadores sostienen que basta con la observación de las partículas obtenidas del raspado superficial de los objetos para tener una noción de las contaminaciones post-deposicionales (Capdepont et al. 2012). Es necesario remarcar que la principal herramienta de control para identificar la contaminación es la comparación de las comunidades florísticas entre las distintas muestras.

El segundo momento de contaminación puede ser durante el tratamiento y/o preservación del material arqueológico. Por ejemplo, lavados o tratamientos previos en los objetos pueden generar incorporación o eliminación de valvas en el material, lo que es necesario conocer bien la historia de conservación del registro arqueológico antes de analizarlo (Robertson 2006; Tuji et al. 2014). Por consiguiente, las comparaciones entre las comunidades de diatomeas de las muestras analizadas, más el conocimiento sobre el contexto arqueológico y de conservación, son prerrequisitos necesarios para que los investigadores generen inferencias con mayor grado de certeza.

Como todo fósil, es necesario entender los problemas tafonómicos que pueden afectar las interpretaciones, ya que las diatomeas pueden presentar cierto deterioro debido a diversos procesos químicos (ej. disolución) y físicos (ej. fragmentación) que afectan sus valvas, generando posibles distorsiones y/o sesgos en las interpretaciones (Hassan 2015). Estos procesos pueden ser generados por condiciones naturales en el momento de su depositación (ej. disolución o erosión en las valvas debido a que se depositan en un medio rico en carbonatos de sodio) o por procesos culturales prehistóricos (ej. la fragmentación de valvas generados durante la manufactura de la cerámica) (Håkansson y Hulthén 1986; Ryves et al. 2009). Incluso las mismas actividades realizadas durante los tratamientos de las muestras puede generar una mayor fragmentación, como es el 
caso del molido de los tiestos de cerámica o el uso de centrifugadora para el lavado de las muestras y obtención de las valvas (Battarbee 1986; Blanco et al. 2008). Estas distorsiones pueden ser controladas e identificadas si el investigador emplea cuidadosas metodologías durante el tratamiento de las muestras y durante la observación de las valvas (ej. estado de las valvas). Estas consideraciones tafonómicas han sido aplicadas principalmente en las reconstrucciones paleoambientales (Blanco et al. 2008; Hassan et al. 2008; Flower y Ryves 2009; Ryves et al. 2009; Hassan 2015), no así en los estudios enfocados a otras problemáticas arqueológicas. Por ejemplo, muchos estudios mencionan la presencia y/o ausencia de diatomeas sin aclarar si se consideran los fragmentos de diatomeas como señal de presencia y/o cuál es la proporción de estos fragmentos en el total de la muestra. Tener una noción de la fragmentación de los taxones (ej porcentaje de valvas enteras y de fragmentos de valvas en una muestra) así como el estado morfológico de las valvas, puede proveer información importante sobre los procesos tafonómicos (De la Fuente 2002; Grana et al. 2014, Lynch et al. 2015).

Esta última cuestión también conlleva a entender los problemas metodológicos que surgen en estas investigaciones. Como mencionamos en un principio, el estudio de las diatomeas nace dentro de la Ficología, por lo tanto, sus metodologías están asociadas a esta disciplina. Esto implica la necesidad por parte de los arqueólogos de adoptarlas, e incluso, en algunos casos, adaptarlas a sus problemáticas. Así, dicha adopción y/o adaptación responderá de manera pertinente a la pregunta de investigación. Esto se hace extensivo también al tipo de muestra que será analizada (sedimentos, raspados, pasta de cerámica, etc.) ya que las densidades de valvas y la conservación de éstas serán muy distintas en cada caso. Por ejemplo, para un análisis paleoambiental se recomienda el tratamiento de $1 \mathrm{gr}$ de sedimento en seco debido a que la sedimentación de valvas en ambientes acuáticos suele ser alta. En cambio en sedimentos arqueológicos es más baja, por lo que debe ser procesada una mayor cantidad de sedimentos para obtener una cantidad mínima de valvas que permitan generar datos cualitativos/cuantitativos válidos.

Un ejemplo que nos debe llevar a la reflexión dentro de esta problemática es el estudio de múltiples microfósiles (diatomeas, fitolitos, polen, espículas, almidones) que propone el uso de una metodología en común para la obtención y análisis de los diversos proxies generando, de este modo, interpretaciones complementarias entre las distintas evidencias. Ahora bien, cabe señalar que este abordaje multiproxy tiene ciertas ventajas (ej. combinación de datos, cantidad de evidencias y tiempo de procesamiento, entre otras) que lo hace atractivo a la hora de ser aplicado en el campo de la Arqueología. No obstante, en muchos casos se advierte que el análisis de las diatomeas queda subsumido o su importancia relegada respecto de las otras evidencias, lo cual conlleva que los datos obtenidos puedan tener algunos sesgos. Esta situación es la que debe llamarnos a la reflexión y por ende se hace necesario tomar ciertas precauciones metodológicas a la hora de afinar las interpretaciones que puedan generar las diatomeas. Por ejemplo, los preparados montados en estos estudios suelen realizarse con medios líquidos o viscosos (ej. aceites de silicona o de inmersión) ya que algunos proxies necesitan ser observados desde una visión en 3D, lo que no es recomendado para las diatomeas por diversos motivos. El primero se debe al bajo índice de refracción (IR) que tiene estos medios de montaje (aceites de silicona IR 1.4; aceite de inmersión IR 1.5). Para observar adecuadamente las valvas de las diatomeas se recomienda el uso de montajes con IR altos $(\sim 1.7)$, como lo tienen las resinas sintéticas Naphrax® o Meltmount ${ }^{\circledR}$ (Julius y Theriot 2010). Pero estas resinas son sólidas, por lo que no serían adecuadas para la observación de otros proxies (fitolitos, polen). Por tanto, la observación en conjunto de los distintos microfósiles dentro de un mismo preparado no sería conveniente. El segundo motivo involucra a los aumentos del microscopio que se suelen usar en estas metodologías. Por lo general, al estar los preparados montados en medios no fijos suelen ser observados con objetivos menores a 1000x. No obstante, las identificaciones y/o determinaciones de especies en diatomeas deben ser realizadas con microscopio de altos aumentos ( $\geq 1000 x)$ ya que es necesario observar bien la ornamentación de las valvas para su identificación (para más detalles ver Round et al. 1999, quienes resumen en general los detalles necesarios para cada género).

Esta precaución sería extensiva incluso para los análisis de presencia/ausencia de valvas ya que existen especies muy pequeñas $(<6 \mu \mathrm{m})$, como algunos géneros de Fragilariaceae que son muy comunes en diversos ambientes (Morales 2001). El empleo de otro tipo de herramienta óptica con aumentos menores no permitiría registrarlas, generando así los resultados con ciertas incertidumbres. Por consiguiente, se vuelve necesario tener en cuenta los requisitos mínimos del microfósil para poder efectuar una identificación precisa. Los estudios multiproxy no están exentos de estos requerimientos, por lo que debieran contemplarse cuáles son los proxies compatibles para combinar en los tratamientos y cuáles deben ser realizados de forma independientes en submuestras paralelas. Esto permitirá poder obtener una información más exacta y con utilidad explicativa.

Por otro lado, es necesario resaltar que a veces suele considerarse la mera presencia de valvas de diatomeas en las muestras como un indicador directo de la presencia de agua. Si bien las diatomeas son indicadoras de humedad, no necesariamente implican la presencia de agua, debido a que existen algunas especies, conocidas como aerófilas, que pueden habitar y sobrevivir sin ningún inconveniente en sustratos (corteza de árboles, rocas, etc.) que reciben 
humedad únicamente de la atmósfera (lluvia, niebla) o también estar en suelos periódicamente secos o con altas fluctuaciones de humedad (Johansen 2010). Por lo tanto, una fuerte abundancia de estas especies podría estar indicando solo condiciones de humedad o resistencia a la desecación y no necesariamente la presencia de agua.

Este punto se relaciona con la cuarta precaución que se debe tener a la hora de interpretar las diatomeas en las muestras: la taxonómica. La determinación de las especies es importante para entender la información ecológica que brindan las diatomeas. Además, como vimos anteriormente, esta determinación puede generar importante información que contribuirá a responder preguntas arqueológicas puntuales (ver los casos aplicados de Beltrán de Heredia Bercero et al. 2006; Solá y Morales 2007; Flores Caballero 2014; Grana et al. 2014).

La taxonomía de las diatomeas está en constante cambio, dado que el empleo de nuevas tecnologías ópticas, como el microscopio electrónico de barrido, han permitido a los taxónomos revisar las especies descriptas en el pasado (Morales 2001). Además, las investigaciones en regiones que tradicionalmente no han sido sistemáticamente estudiadas, ha brindado a los taxónomos material original con especies endémicas para realizar nuevas determinaciones y generar más datos ecológicos (Maidana y Herbst 1989). La Arqueología es una fuente significativa para brindar a los diatomólogos material fósil con posibles nuevos taxones, generando así una retroalimentación interdisciplinaria.

\section{Conclusión}

La recopilación bibliográfica presentada en este trabajo permite remarcar que el análisis de diatomeas en las investigaciones arqueológicas tiene un importante y amplio rango de aplicaciones, que puede abarcar diversas temáticas con diferentes escalas espaciales y temporales. El valor del dato de las diatomeas dependerá de la pregunta del investigador y de cuánta equidad en lo que respecta a calidad de información se quiere obtener en relación a los distintos proxies analizados. Además su aplicación, como cualquier otro bioindicador, requiere que el investigador tenga un buen conocimiento de su biología, su ecología y su sistemática para evitar posibles sesgos en sus interpretaciones. Para ello, es necesario que exista un compromiso por parte del arqueólogo en conocer las limitaciones y los requisitos mínimos de este proxy.

Las temáticas más tradicionales que han empleado el análisis de las diatomeas en sus investigaciones han sido las ambientales y en artefactos arqueológicos como las cerámicas, sin embargo, hemos visto que su aplicación puede ser aún mucho más amplia. No obstante, no se puede afirmar que esas aplicaciones agoten todo el rango posible, ya que sólo nuevas preguntas, que conlleven a trabajos interdisciplinarios entre los arqueólogos y los diatomólogos, permitirán explorar y enriquecer los resultados, e incluso generar nuevas y potenciales temáticas.

San Fernando del Valle de Catamarca, 10 de mayo 2017

\section{Agradecimientos}

Quiero agradecer a la Dra. Jennifer Grant por brindar una lectura crítica del manuscrito. A la Dra. Patricia Escola y la Lic. Verónica Zucarelli por sus valiosos comentarios en algunos temas específicos. A los evaluadores anónimos por las sugerencias que enriquecieron el trabajo. Y por último, quiero agradecer y dedicar este trabajo a la Dra. Nora I. Maidana por estar siempre predispuesta a enseñarme sobre el mundo de las diatomeas y a crear juntas puentes de comunicación entre la Arqueología y la Diatomología.

\section{Bibliografía}

Alhonen, P., J. Kokkonen, H. Matiskainen \& A. Vuorinen. 1980. Applications of AAS and diatom analysis and stylistic studies of Finnish Subneolithic pottery. Bulletin of the Geological Society of Finland, 52: 193-206

Ashley, G., J. Tactikos \& R. Owen. 2009. Hominin use of springs and wetlands: Paleoclimate and archaeological records from Olduvai Gorge ( 1.79-1.74 Ma). Palaeogeography, Palaeoclimatology, Palaeoecology, $272(1-2): 1-16$.

Babot, P. 2004. "Tecnología y Utilización de Artefactos de Molienda en el Noroeste Prehispánico". Facultad de Ciencias Naturales e Instituto Miguel Lillo, Universidad Nacional de Tucumán. San Miguel de Tucumán, pp. 280. Tesis de postgrado.

Bathurst, R., D. Zori \& J. Byock. 2010. Diatoms as bioindicators of site use: locating turf structures from the Viking Age. Journal of Archaeological Science, 37(11): 2920-2928.

Battarbee, R. W., 1986. Diatom analysis. En: Berglund, B. E. (ed.) Handbook of Holocene Palaeoecology and Palaeohydrology. Pp. 527-570 Chichester, Wiley.

Battarbee, R.1988. The Use of Diatom Analysis in Archaeology: A Review. Journal of Archaeological Science, 15: 621-644.

Belmar, C., X. Albornoz, S. Alfaro, F. Meneses, C. Carrasco, L. Quiroz \& T. Planella. 2016. Reconstruyendo las prácticas fumatorias del sitio La Granja (130 A.C 1000 D.C., Valle del Río Cachapoal, VI Región, Chile Central) a partir de los Microfósiles. Chungará, Revista de Antropología Chilena, 48(1): 53-72. 
Beltran de Heredia Bercero, J., M. Comas Solá, E. Gurri Costa \& J. Tresserras. 2006. Noves aportacions sobre la producció de vi a la laietània Romana: Installacions Vinícoles i anàlisis de residus. Quarhis, 2: 92-107.

Benes, J., J. KaStovský, R. KoCárová, P. KoCár, K. KubeCková, P. Pokorný \& P. Starec 2002. Archaeobotany of the Old Prague Town defence system, Czech Republic: Archaeology, macro-remains, pollen, and diatoms. Vegetation History and Archaeobotany, 11(1-2): 107-119.

Blanco, S., Álvarez, I., \& C. Cejudo. 2008. A test on different aspects of diatom processing techniques. Journal of Applied Phycology, 20(4): 445-450.

Blasi A., C. Castiñeira, L. del Puerto, H. Inda, R. Bracco \& F. García-Rodríguez. 2005. Sedimentación Holocena en los Bañados de Santa Teresa y el Registro Arqueológico, Planicie Costera de Departamento de Rocha, Uruguay". En: Actas del XVI Congreso Geológico Argentino. Tomo IV pp. 289-297.2005. Asociación Geológica Argentina. La Plata.

Bolívar, F. \& P. Sánchez-Castillo. 1997. Biomineralization Processes in the Fountains of the Alhambra, Granada, Spain. International Biodeterioration \& Biodegradation, 40 (2-4): 205-215.

Bonomo, M., A. Zucol, B. Gutiérrez Téllez, A. Coradeghini \& M. Vigna. 2009. Late Holocene palaeoenvironments of the Nutria Mansa 1 archaeological site, Argentina. Journal Paleolimnology, 41: 273-296.

Bonomo, M., M. Colobi \& N. Mazzia. 2012. Análisis de residuos orgánicos y microfósiles silíceos de la "cuchara" de cerámica del sitio arqueológico Cerro Tapera Vázquez (Parque Nacional Pre-Delta, Argentina. Revista do Museu de Arqueologia e Etnologia, 22: 31-50.

Bradshaw, E., A. Nielsen \& N. Anderson. 2006. Using diatoms to assess the impacts of prehistoric, preindustrial and modern land-use on Danish lakes. Regional Environmental Change, 6: 17-24.

Cameron, N. 2007. Diatom Methods/Use in Archaeology. En Elias, S. \& C. Mock (Eds.) Encyclopedia of Quaternary Science, pp. 523-529. Amsterdam, Elsevier.

Capdepont, I. \& M. Bonomo. 2010. Análisis petrográfico de material cerámico del Delta del Paraná. Anales de Arqueología Y Etnología, 65-67: 161-181.

Capdepont, I., Inda, H., \& L. del Puerto. 2002. Caracterización tecnológica y funcional del material cerámico arqueológico de la cuenca de la Laguna de Castillos (Rocha-Uruguay). En D. L. Mazzanti, M. A. Berón, \& F. W. Oliva (Eds.), Del mar a los salitrales. Diez mil años de historia pampeana en el umbral del tercer milenio. pp. (pp. 41-50). Mar del Plata: Facultad de Humanidades, UNMdP/SAA.

Caria, M., J. Gomez Augier, H. Cruz \& J. Zapatiel. 2011. Aportes a la construcción de la variabilidad material de un sitio arqueológico en el piedemonte oriental de cumbres calchaquíes- Tucumán. Comechingonia, 14 (1): 133-154

Carlie, A., C. Arcini, H. Druid \& J. Risberg. 2015. Archaeology, forensics and the death of a child in Late Neolithic Sweden. Antiquity, 88 (342): 1148-1163.

Castiñeira Latorre, C. 2009. Aspectos de la colonización humana prehistórica del Noroeste del Uruguay. Arqueología, 15: 241-245.

Castiñeira, C., A. Blasi, G. Politis \& M. Bonomo. 2013. The origin and construction of pre-Hispanic mounds in the Upper Delta of the Paraná River (Argentina). Archaeological and Anthropological Sciences, 5 (1): 37-57.

Coil, J., A. Korstanje, S. Archer \& C. Hastorf. 2003. Laboratory goals and considerations for multiple microfossil extraction in archaeology. Journal of Archaeological Science, 30(8): 991-1008.

Colobig, M. \& F. Ottalagano. 2016. Estudio arqueobotánico de los residuos orgánicos adheridos en alfarerías prehispánicas de la cuenca del Paraná medio. Arqueología, 22 (1): 193-210.

Colobig, M. D. L. M., Zucol, A. F., Mazzanti, D. L., \& Passeggi, E. 2016. Registros biosilíceos en sitios arqueológicos de las sierras de Tandilia Oriental, Argentina (Pleistoceno Tardío-Holoceno Tardío): Consideraciones paleoambientales. Revista Museo Argentino de Ciencias Naturales, 18(1): 39-52.

Cooper, S., Gaiser, E. \& A. Wachnicka 2010. Estuarine paleoenvironmental reconstructions using diatoms. En: E. Stoermer \& J. Smol (Eds.) The Diatoms, applications for Environmental and Earth Sciences, (pp. 324-345). Londres, Cambridge University Press.

De la Fuente, G. 2002. Diatomological analysis (Provenance) application in Archaeological ceramics: an experimental approach. En: E. Jerem \& K. Biró (Eds.), Archaeometry 98: Proceedings of the 31 st Symposium, Budapest, April 26-May 3 1998. Volumen II, pp 501-511. BAR International Series. Archaeopress. Oxford.

Denhman, T., K. Sniderman, K. Saunders, B. Winsborough \& A. Pierret. 2009. Contiguous multi-proxy analyses (X-radiography, diatom, pollen and micro- charcoal) of Holocene archaeological features at Kuk Swamp, Upper Wahgi valley, Papua New Guinea. Geoarchaeology: An 
International Journal 24 (6): 715-742.

Dubois, C. 2006. Dinámica fluvial, paleoambientes y ocupaciones humanas en la localidad arqueológica Paso Otero, río Quequén Grande, Provincia de Buenos Aires. Intersecciones en Antropología, 7: 109-127.

Dudgeon, J. \& M. Tromp. 2014. Diet, Geography and Drinking Water in Polynesia: Microfossil Research from Archaeological Human Dental Calculus, Rapa Nui (Easter Island). International Journal of Osteoarchaeology, 24 (5): 634-648

Fernández, M. \& M. Salemme. 2012. Diatom analysis in Santa Cruz central Massif (Patagonia, Argentina): preliminary results. Current Research in the Pleistocene, 29 (special edition): 191-196

Fernández, M. 2012. "Los paleoambientes de Patagonia meridional, Tierra del Fuego e Islas de los Estados en los tiempos de las primeras ocupaciones humanas. Estudio basado en el análisis de diatomeas". Facultad de Ciencia Naturales y Museo. Universidad Nacional de La Plata, Argentina, pp. 226 Tesis de postgrado. FCNyM. UNLP.

Flores Caballero, K. A. 2014. "Determinación de Organismos Biológicos en Coprolitos de Pobladores Precolombinos de la Ciudad Pesquera de Áspero" Facultad de Ciencias Biológicas. Universidad Nacional Mayor de San Marcos, Perú. Tesis de Grado.

Flower R. 2006. Diatoms in ancient building materials: application of diatom analysis to Egyptian mud bricks. Nova Hedwig Beih 130:245-264.

Flower, R., \& D. Ryves. 2009. Diatom preservation: differential preservation of sedimentary diatoms in two saline lakes. Acta Botanica Croatia, 68(2): 381-399.

Frenguelli, J. 1940. El ambiente geográfico. Relaciones 2: $13-38$

Fugassa, M., M. González Sagrario, O. Beltrame \& N. Sardella. 2011. Rotíferos y otros invertebrados en sedimentos arqueológicos de la colección del Museo Regional Monseñor Fagnano, Rio Grande, Tierra Del Fuego, Argentina. Revista Argentina de Antropología Biológica, 13(1): 55-60.

Gasparotti, L. \& C. Haros. 2015. Contenedores y contenidos. Explorando el consumo de vegetales durante el Período Tardío (Antofagasta de la Sierra, Provincia de Catamarca). Revista Del Museo de Antropología, 8(2): 47-62.

Gearey, B., A. Fletcher, W. Fletcher, I. Boomer, D. Keen, J. Reed \& E. Tetlow. 2011. From site to landscape: Assessing the value of geoarchaeological data in understanding the archaeological record of Domuztepe, Eastern Mediterranean, Turkey. American Journal of Archaeology, 115(3): 465-482.

Gibson, A. 1986. Diatom analysis of clays and Late Neolithic Pottery from the Milled diatom analysis of clays and Late Neolithic Pottery from the Milfield Basin, Northumberland. Proceedings of the Prehistoric Society, 52: 89-103.

Grana L., P. Tchilinguirian, S. Hoscman, P. Escola \& N. I. Maidana. 2016. Palaeoenvironmental changes, river fluctuation and human settlement at 7000-3000 cal. yr BP in a highland desert (Antofagasta de la Sierra, Southern Puna, Argentina). Geoarchaeology, an International Journal, 31(5): 412-433.

Grana, L. 2013. “Arqueología y Paleoambiente: Dinámica Cultural y Cambio Ambiental en Sociedades Complejas de la Puna Meridional Argentina". Facultad de Filosofía y Letras, Universidad de Buenos Aires, Argentina, pp. 353. Tesis postgrado.

Grana, L., M. Cohen \& N. Maidana. 2014. Methodological proposal to identify irrigation canals using diatom analyisis as a biomarker: Peñas Coloradas (Antofagasta de la Sierra, Southern Argentine Puna). Kligmann, D.M., Morales, M.R. (Eds.), Physical, Chemical and Biological Markers in Argentine Archaeology: Theory, Methods and Applications, pp. 73-86. BAR International Series. Archaeopress. Oxford.

Gutiérrez, M.A., Martínez, G., Luchsinger, H., Grill, S., Zucol, A.F., Hassan, G.S., Barros, M.P., Kaufmann, C.A. \& Álvarez, M.C. 2011. Paleoenvironments in the Paso Otero locality during Late Pleistocene-Holocene (Pampean region, Argentina): An interdisciplinary approach. Quaternary International 245: 37-47

Hadley, K., M. Douglas, R. McGhee, J. Blais \& J. Smol. 2010. Ecological influences of Thule Inuit whalers on high Arctic pond ecosystems: A comparative paleolimnological study from Bathurst Island (Nunavut, Canada). Journal of Paleolimnology, 44(1): 85-93.

Håkansson, H. \& B. Hulthén. 1986. On the dissolution of pottery for diatom studies. Norwegian Archaeological Review, 19 (1): 34-38

Hákansson, H. \& B. Hulthén. 1988. Identification of diatoms in Neolithic Pottery. Diatom Research, 3: 39-45.

Hassan, G. 2012. Diatomeas del Holoceno tardío en sitios arqueológicos del sistema de Tandilia. En: D. L. Mazzanti (ed.), Cazadores-recolectores de las Sierras de Tandilia Oriental. El Holoceno tardío. Publicación Especial 3. Laboratorio de Arqueología de la Universidad Nacional de Mar del Plata. Mar del Plata. 
Hassan, G. 2015. On the benefits of being redundant: low compositional fidelity of diatom death assemblages does not hamper the preservation of environmental gradients in shallow lakes. Paleobiology, 41 (1): 154-173.

Hassan, G.S., Espinosa, M.A., Isla, F.I. 2008. Fidelity of dead diatom assemblages in estuarine sediments: how much environmental information is preserved? Palaios 23: $112-120$.

Horrocks, M., M. Jones, S. Nichol \& D. Sutton. 2002. Wetland Microfossils in Soil: Implications for the Study of Land Use on Archaeological Landscapes. Environmental Archaeology, 7: 101-106

Inda, H., Garcia-Rodriguez, F., Del Puerto, L., Figueira, R., \& S. Stutz. 2016. Discriminating between natural and human induced shifts in a shallow coastal lagoon: a multidisciplinary approach. Anthropocene, 16: 1-15

Johansen, J. 2010. Diatoms in aerial habitats. En: Stoermer, E. F. and Smol J. P. (Eds.). The Diatoms: Applications for the Environmental and Earth Sciences, $2^{\circ}$ edición pp. 264- 276.Cambridge University Press, Cambridge.

Juan-Tresserras, J. 2000. La cerveza: Un producto de consumo básico entre comunidades Ibéricas del N.E. Peninsular. SAGVNTVM-PLAV-Papeles del Laboratorio de Arqueología de Valencia-Extra, 3: 139-145.

Juggins, S. \& Cameron, N., 2010. Diatoms and archaeology. The Diatoms: Applications for the Environmental and Earth Sciences, $2^{\circ}$ edición pp. 389-401. Cambridge University Press, Cambridge.

Julius, M.L. \& E.C. Theriot 2010. The diatoms: a primer. En: E. Stoermer \& J. Smol (Eds.) The Diatoms, applications for Environmental and Earth Sciences (pp. 8-22). Londres, Cambridge University Press.

Kligmann, D. \& M. Calderari. 2012. Diatoms and ceramic provenance: A cautionary tale. Archaeometry, 54(1): 129-143.

Kligmann, D. 2009. Procesos de formación de sitios arqueológicos: tres casos de estudio en la Puna meridional catamarqueña argentina, pp. 327. British Archaeological Reports (BAR) International Series 1949, Archaeopress, Oxford.

Kligmann, D. M., Ratto, N., \& Maidana, N. I. 2013. Microarqueología y procesos de formación del registro: análisis mineralógicos y diatomológicos del sitio $\mathrm{El}$ Zorro (Puna transicional, departamento de Tinogasta, Catamarca). M. Ramos (Ed.), Arqueometría argentina: estudios pluridisciplinarios. 113-136, Aspha. Buenos Aires.

Korstanje, M. A. \& P. Cuenya. 2010. Ancient agriculture and domestic activities: a contextual approach studying silica phytoliths and other microfossils in soils. Environmental Archaeology, 15(1): 43-63.

Korstanje, M. A. 2005. “La Organización del Trabajo en Torno a la Producción de Alimentos, en Sociedades Agropastoriles Formativas (Pcia. de Catamarca, Rep. Argentina)". Facultad de Ciencias Naturales e Inst. M. Lillo. Universidad Nacional de Tucumán, Argentina. Tesis de postgrado.

Korstanje, M.A \&P. Babot (eds) 2008. Matices Interdisciplinarios en Estudios Fitolíticos y de Otros Microfósiles. Pp 245. BAR International Series S1870, Oxford

Lozano-García, M., M. Caballero \& B. Ortega. 2007. Evidencia del Impacto Humano y Cambio Climático Natural en la Región de los Tuxtlas: Un enfoque multidisciplinario. Revista Especializada en Ciencias Químico-Biológicas, 10(2): 58-64.

Lynch, V., M. Fernández \& L. Miotti. 2015. Estudio experimental sobre la dinámica postdepositacional en rastros de uso: integridad artefactual del componente 1 de Cueva Maripe (Santa Cruz, Argentina). Magallania, 43(1): 211-229.

Maidana, N. I. 2013. El test de diatomeas en el diagnóstico de muerte por sumersión. ACTA NOVA, 6(1-2): 70-81.

Maidana, N.I. \& N. Herbst. 1989. Diatomeas (Bacillariophyceae) de la Laguna Nostra (Cumbres Calchaquíes), provincia de Tucumán, República Argentina. Darwiniana 29 (1-4): 63-76.

Mannion, A.M. 1987. Fossil Diatoms and Their Significance in Archaeological Research. Oxford Journal of Archaeology, 6 (2): 131-147.

Martínez Macchiavello, J., G. De La Fuente \& N. Ratto 1999. La utilización del análisis de diatomeas (Bacillariophyta) en la investigación arqueológica: una perspectiva interdisciplinaria. Arqueología 9: 49-69

Martínez, G., D. Mazzanti, C. Quintana, A. Zucol, M. Colobig, G. Hassan \& E. Passeggi. 2014. Geoarchaeological and Paleoenvironmental context of the human settlement in the Eastern Tandilia Range, Argentina. Quaternary International, 299: 23-37.

Meltzer, D. \& M. Collins. 1987. Prehistoric water wells on the Southern High Plains: clues to Altithermal climate. Journal of Field Archaeology, 14(1): 9-28.

Morales, E. A. 2001. Morphological studies in selected fragilarioid diatoms (Bacillariophyceae) from Connecticut waters, USA. Proceedings Academy Natural Sciences 
Philadelphia 151: 39- 54.

Morales, M. R. 2011. Arqueología ambiental del Holoceno Temprano y Medio en la Puna Seca Argentina. Modelos paleoambientales multi-escalas y sus implicancias para la Arqueología de Cazadores-Recolectores. Pp. 241 British Archaeological Reports (BAR) International Series 15. Archaeopress, Oxford.

Neely, J., S. Caran, B. Winsborough, F. Ramirez Sorensen \& S. Valastro. 1995. An early holocene hand-dug water well in the Tehuacan Valley of Puebla, Mexico. Current Research in the Pleistocene, 12: 38-40.

Obando, L., S. Jiménez \& S. Kussmaul. 2011. Estudio petrográfico de cerámicas Mayas, Clásico Tardío (600-900 D.C.), Chinikihá, Chiapas, México. Revista Geológica de América Central, 44:101-118.

Ogalde J., B. Arriaza, C. Paipa, P. Leyton, M. CamposVallette, N. Lara, C. Salas \& P. Tapia. 2015. Multiinstrumental characterization of two red pigments in funerary archaeological contexts from Northern Chile. Interciencia, 40 (12): 875-880

Ognjanova-Rumenova, N. 2008. Palaeoenvironment and archaeology: the use of diatom analysis in archaeology. En: Kostov, R., B. Gaydarska y M. Gurova (eds.) Geoarchaeology and archaeomineralogy, Proceedings of International Conference 29-30 October 2008, pp291-294. Publ. house St. Iv. Rilski, Sofia.

Ognjanova-Rumenova, N. y D. Zaprjanova. 1998. Siliceous microfossil stratigraphy of sediment profile " $F$ " connected with archaeological excavation in coastal wetlands in the Bay of Sozopol (Bulgarian Black Sea coast). Part I: Taxonomical documentation, diatom biostratigraphy and ecological analysis of the diatom flora. Phytologia Balcanica, 4 (1-2):3-17

Owen, R., J. Barthelme, R. Renaut \& A. Vincens. 1983. Palaeolimnology and Archaeology of Holocene deposits north-east of Lake Turkana, Kenya. Nature 298: 523-529.

Patrick, R. 1938. The Occurrence of Flints and Extinct Animals in Pluvial Deposits near Clovis, New Mexico. Part $\mathrm{V}$ : Diatom Evidence from the Mammoth Pit. Proceedings of the Academy of Natural Sciences of Philadelphia, 90: 15-24.

Peraza Zurita, Y., G. Cultrone, P. Sánchez Castillo, E. Sebastión \& F. Bolívar. 2005. Microalgae associated with deteriorated stonework of the fountain of Bibataubín in Granada, Spain. International Biodeterioration \& Biodegradation, 55: 55-61.

Planella, M. T., Belmar, C. A., Quiroz, L. \& Estévez, D. 2012. Propuesta Integradora para un Estudio del Uso de
Plantas con Propiedades Psicoactivas en Pipas del Período Alfarero Temprano y sus Implicancias Sociales. Revista de Antropología, 21(1): 93-119.

Ramírez Ríos, D.C., Otálora Cascante, A.R., \& Parra Giraldo, R. 2008. Identificación de Fitolitos en el cálculo dental de individuos prehispánicos del Valle del Río Cauca, Colombia. En Korstanje, A. \& M. del P. Babot (Eds.) Matices Interdisciplinarios en Estudios Fitolíticos y de Otros Microfósiles, pp. 209-217. British Archaeological Reports, Oxoford.

Robertson, G. 2006. Diatoms and sponge spicules as indicators of contamination on utilised backed artefacts from Turtle Rock, Central Queensland Highlands. En: S. Ulm \& I. Lilley (Eds.), An Archaeological Life: Papers in Honour of Jay Hall. Research Report Series 7. Pp. 125140. University of Queensland, Brisbane.

Round F., R. Crawford \& D. Mann. 1990. The diatoms: biology and morphology of the genera. Pp751. Cambridge University Press, New York.

Ryves, D. B., Battarbee, R. W. \& S. C. Fritz. 2009. The dilemma of disappearing diatoms: Incorporating diatom dissolution data into palaeoenvironmental modelling and reconstruction. Quaternary Science Reviews, 28(1-2): 120-136.

Sánchez, J., M. Colobig, A. Zucol, G. Politis, M. Bonomo \& C. Castiñeira. 2013. Primeros resultados sobre el uso prehispánico de los vegetales en el sitio arqueológico Los Tres Cerros 1 (Victoria, Entre Ríos, Argentina): análisis del registro biosilíceo. Darwiniana, 1(2): 201-219.

Smol, J. 2009. Pollution of lakes and rivers: a paleoenvironmental perspective. Pp396. Arnold and Oxford University Press, Londres.

Solá, P. \& M. Morales. 2007. Caracterización petrográfica y biológica de un tiesto cerámico estilo "incaico" hallado en Susques (Jujuy). Intersecciones En Antropología, 8: 361-364.

Tchilinguirian, P. \& D. Olivera. 2010. Evolution and Human Land Management during the Holocene in southern altiplano desert, Argentina (26'S). Zdruli,P., M. Pagliai, S. Capur y A. Faz Cano (Eds) Land Degradation and Desertification: assessment, mitigation and remediation, Pp. 591-604, Springer, Holanda.

Trombold, C. \& I. Israde-Alcantara. 2005. Paleoenvironment and plant cultivation on terraces at La Quemada, Zacatecas, Mexico: The pollen, phytolith and diatom evidence. Journal of Archaeological Science, 32(3): 341-353.

Tuji, A., A. Marsh, M. Altaweel, C. Watanabe \& J. Taylor. 
2014. Diatom Analysis of Cuneiform Tablets Housed in the British Museum. Bulletin of the National Museum of Nature and Science. Series B, 40 (3): 101-106.

Vargas Afonso, J. 2007. Silicofitolitos y gránulos de almidón en cálculos dentales de antiguas poblaciones de Tenerife: Propuesta para la ampliación del estudio de la dieta y alimentación históricas. Revista Tabona, 15: 143-162.

Vásquez Sánchez, V., F. Jordán Régulo \& T. Tham. 2014. Almidones antiguos del cálculo dental de un entierro mochica de la Huaca Cao Viejo, complejo arqueológico El Brujo, costa norte del Perú. Revista Archaeobios, 8(1): 6-16.

Villagran, X., P. Deblasis \& P. Giannini. 2009. Nota breve Primeros estudios micromorfológicos en sambaquís brasileños (sitio Jabuticabeira II, Estado de Santa Catarina). Intersecciones En Antropología, 10: 359-364.

Vouilloud. A. 2003. Catálogo de diatomeas continentales y marinas de Argentina. Pp 308. Asociación Argentina de Ficología, La Plata.

Wallis, N., A. Cordell, K. Deagan \& M. Sullivan. 2014. Inter-ethnic social interactions in 16th century La Florida: sourcing pottery using siliceous microfossils. Journal of Archaeological Science, 43: 127-140.
Weide, M.D, S.C. Fritz, C.A. Hastorf, M.C. Bruno, P.A. Baker, S. Guedron \& W. Salenbien. 2017. A 6000 yr diatom record of mid- to late Holocene fluctuations in the level of Lago Wiñaymarca, Lake Titicaca (Peru/Bolivia). Quaternary Research, 88 (2): 179-192.

Winsborough, B., S. Caran, J. Neely \& S. Valastro. 1996. Calcified Microbial Mats Date Prehistoric CanalsRadiocarbon Assay of Organic Extracts from Travertine. Geoarchaeology: An International Journal, 11(1): 37-50.

Wood, J. \& J. Wilmshurst. 2016. A protocol for subsampling Late Quaternary coprolites for multi-proxy analysis. Quaternary Science Reviews, 138: 1-5.

Zucol, A., G. Figueroa \& M. Colobig. 2012. Estudio de microrrestos silíceos en sistemas de aterrazamiento del primer milenio DC en el Valle de Ambato (Andes del sur), Catamarca, Argentina. Intersecciones En Antropología, 13: 163-179.

Zucol, A., M. Brea \& D.L. Mazzanti. 2008. Análisis de restos orgánicos presentes en cerámicas arqueológicas de las Sierras de Tandilia (provincia de Buenos Aires, Argentina). En Korstanje, A. \& M. del P. Babot (Eds.) Matices Interdisciplinarios en Estudios Fitolíticos y de Otros Microfósiles, pp. 201-208. British Archaeological Reports, Oxoford. 